\title{
Patient Specific Simulated Dynamics following TKA Correlate with Patient Reported Outcomes
}

\author{
Joshua Twiggs $\mathrm{BE}^{1,2 *}$, Willy Theodore $\mathrm{BE}^{1}$, Andrew Ruys $\mathrm{PhD}^{2}$, Justin Roe \\ $\mathrm{MD}^{3}$, David Dickison $\mathrm{MD}^{4}$, Brett Fritsch $\mathrm{MD}^{5}$, Brad Miles $\mathrm{PhD}^{1}$ \\ ${ }^{1 * C o r r e s p o n d i n g ~ A u t h o r / 360 ~ K n e e ~ S y s t e m s, ~ S y d n e y, ~ 2073, ~ A u s t r a l i a ~}$ \\ ${ }^{2}$ Department of Biomedical Engineering, University of Sydney, Sydney, 2050, Australia \\ ${ }^{3}$ North Sydney Orthopaedic and Sports Medicine Centre, The Mater Hospital, North Sydney, 2060, \\ Australia \\ ${ }^{4}$ Peninsula Orthopaedics, Sydney, 2099, Australia \\ ${ }^{5}$ Sydney Orthopaedic Research Institute, Sydney, 2067, Australia \\ joshua@kneesystems.com
}

\section{INTRODUCTION}

Total Knee Arthroplasty (TKA) is an established procedure for improving pain and restoring function to patients who have osteoarthritis (OA). However, around $20 \%$ of recipients remain dissatisfied with the outcome. [1] Major reasons for post-operative dissatisfaction include recurrent pain and functional impairment. [2, 3]

Increasingly, focus has been placed on Patient Reported Outcome Measures (PROMS) as both a research tool and clinical measure of outcome following TKA. [4] Component alignment has been shown to have some impact on postoperative PROMS, [5] most significantly femoral and tibial component rotation. [6-8] However, as there are numerous alignment strategies that can lead to positive patient outcomes, $[9,10]$ it would appear that component alignment does not explain the full scope of patient variation in outcomes.

These seemingly contradictory observations might be better understood if it were possible to look at an individual patients' dynamic characteristics in similarly designed observational studies. Studies have previously shown post-operative joint kinematics to be significantly different from preoperative kinematics following TKA. [11, 12] Furthermore, studies have shown that joint kinematics vary with component alignment, sometimes in ways that are reproducible between patients. [13-16] Other alignment parameters have not been shown to have clearly identifiable reproducible relationships with kinematic outcomes, with evidence that patient specific characteristics are a factor. [17, 18] 
Dynamic knee computer simulations have been developed which allow the impact of patient and surgical factors on kinematics following TKA to be observed. [19, 20] A major advantage of these simulations is the relative ease with which they can be deployed relative to alternative assessments of TKR dynamics like cadaveric testing, In vivo Fluoroscopy or gait lab assessments. [21] These simulations are increasingly being used to study the impact of component placement variation [22-24] and are able to incorporate patient specific elements with the use of readily available diagnostic radiology such as Computed Tomography (CT) scans. [25, 26]

Simulations incorporating patient specific elements might be a mechanism for uncovering an underlying link between postoperative TKA dynamics and PROMS that has so far been observed in relationships with component alignment, but not directly measured. This study aimed to determine if the output dynamics derived from consideration of both postoperative component alignment and patient specific musculoskeletal modelling correlated with Patient Reported Outcomes.

\section{MATERIALS AND METHODS}

A musculoskeletal computational simulation of an Oxford Knee Rig was developed. The simulation uses post-TKA CT scan inputs of all relevant landmarks, bones and registered component positions. This model has been previously validated against a series of 8 cadaveric knees. [27] A series of 116 patients were enrolled into the ‘The Joint Dynamics Registry' which includes post-operative CT scans of TKA patients (Bellberry Human Research Ethics Committee, approval number 2012-03-710). All patients received a cruciate retaining TKA using a common implant system (Triathlon, Stryker, Michigan, U.S.A). Surgeries were performed by one of two surgeons and all surgeries were mechanically aligned. Patients also answered a Knee injury and Osteoarthritis Outcome Score (KOOS) at least 1 year after their operation. These patients' CT scans were reconstructed into 3D models and anatomical landmarks and simulated. The results were compared to the KOOS scores and any relationships ascertained.

The simulation replicated a deep knee bend performed in an Oxford Knee Rig. The Oxford Knee Rig allows 6 degree of freedom, with the ankle modelled possessing all 3 rotational degrees of freedom, while the hip does not allow rotation but does vertically translate. [28] The simulation was modelled using ADAMS software (MSC Software, Newport Beach, California). The simulation treats the femoral, tibial and patella components as rigid bodies in contact. A flexion controller drove the simulation through a 10 second simulation cycle after model set up. The first 5 seconds consisted of a deep knee bend to $140^{\circ}$ of flexion, driven by vertical motion of the hip attachment. The last five seconds consisted of extension of the knee to $5^{\circ}$ of flexion, which was driven by a single line of action through the quadriceps tendon.

Each model was assembled from CT scan segmentations of patient geometry using ScanIP segmentation software (Simpleware, Exeter, UK). CT scans were taken at $2 \mathrm{~mm}$ slice thickness, with the other axial thicknesses varying but all less than $2 \mathrm{~mm}$. Biomedical engineers landmarked the femoral, tibial and patella bones. Attachment and insertion sites for the lateral collateral ligament (LCL), medial collateral ligament (MCL), posterior cruciate ligament (PCL), quadriceps tendon and patella tendon were identified. The LCL was modelled as a single bundle from the lateral epicondyle of the femur. The MCL was considered to consist of anterior and posterior bundles, with their origin differentiated anteriorly and posteriorly from the medial epicondyle of the femur. Similarly, the PCL was modelled 
as an anterior and posterior bundle and was differentiated at its origin on the femur. All ligaments were modelled as linear springs, example Figure 1.

The femoral and hip centres were landmarked to define the mechanical axis of the femur. The lateral and medial malleoli were captured to define the tibial mechanical axis, which was defined from the midpoint of these two landmarks to the midpoint of the medial 1/3 of the tubercle and PCL insertion. Rotationally, the tibial anterior-posterior axis was defined along these two points, while the Transepicondylar Axis (TEA) from the medial sulcus to the lateral epicondyle was used for the femur. A patella superior-inferior axis was constructed from superior and inferior points of the patella bone. A full reference frame was defined from a rotational anterior-posterior axis perpendicular to the plane created by these two points and the medial edge of the patella bone.

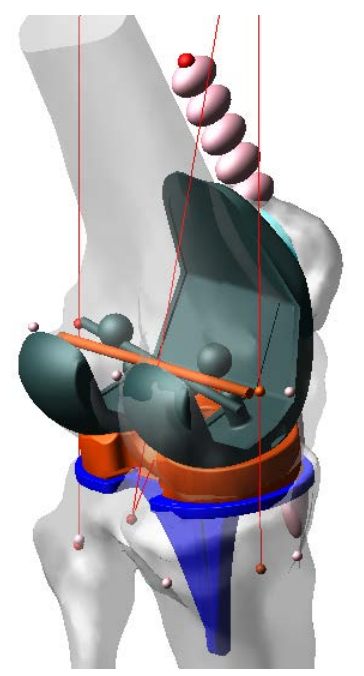

Figure 1: The simulation in extension cycle, with both PCL bundles, the LCL and MCL posterior bundle all actively strained.

The 3D Implant geometries were reverse engineered from laser scans of definitive prosthesis. Implants were registered to the CT scan using the $+\mathrm{CAD}$ module within ScanIP, with the reconstructed results appearing as shown in Figure 2.

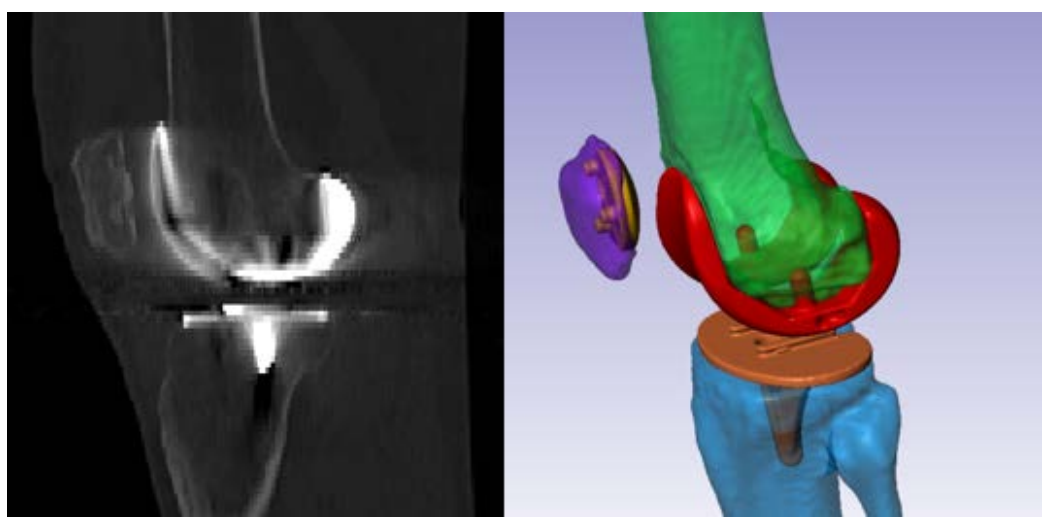

Figure 2: Registration of implant geometry to the CT scan of postoperative TKA patients 
Varus/Valgus alignment and Internal/External Rotation throughout the full flexion cycle were extracted for each simulation as per Grood \& Suntay's joint coordinate system, with the measurements recorded in the tibial reference frame. [29] Femoral rollback was recorded as the antero-posterior translation of the midpoint of the TEA relative to the tibial insert. The quadriceps force required to drive the extension motion was also extracted. Patella tilt and medial-lateral shift were also extracted, with tilt and shift measured relative to the mechanical axis and TEA of the femur respectively. Values of each of these parameters at $10^{\circ}, 45^{\circ}$ and $90^{\circ}$ degrees of flexion and the differences between each of these values were tabulated.

Post-operative KOOS capture was conducted at least 1 year following surgery following surgery. Patients were interviewed individually and were asked to consider the previous seven days as a time frame. Linear spearman's correlations between the KOOS Pain and Symptoms subscores (scores out of 100 , with positive values being a better outcome) and each of the kinematic parameters were determined. T-tests between the lowest $20 \%$ ( 19 , scores $<60$ ) and the remaining $80 \%$ ( 77 , scores $>60$ ) were performed for each linearly correlated relationship to confirm differentiation of patients with a poorer outcome from those performing better. Non-linear relationships were investigated using Classification And Regression Trees (CART) constrained to whole unit increments, and the resultant groups created were t-tested for difference. Significance was set to 0.05 .

\section{RESULTS}

A statistically significant negative correlation $(-0.339, \mathrm{p}<0.001)$ was found between the postoperative KOOS Symptoms score and the rollback occurring from $10^{\circ}$ to $45^{\circ}$ flexion. Likewise, a significant negative correlation was found between the quadriceps force at $45^{\circ}$ of flexion and the postoperative KOOS Symptoms score $(-0.229, \mathrm{p}=0.025)$. Patella lateral tilt negatively correlated at all three flexion points with the Symptoms score $\left(-0.263, \mathrm{p}=0.009\right.$ at $10^{\circ}$ flexion, $-0.282, \mathrm{p}=0.005$ at $45^{\circ}$ flexion and 0.262 , $p=0.010$ at $90^{\circ}$ flexion). Patella lateral shift at $90^{\circ}$ flexion had a significant positive relationship (0.254, $\mathrm{p}=0.012)$. When segmenting the population into the lowest $20 \%(19 / 96)$ and t-testing the two groups formed, statistically significant differences were found for rollback from $10^{\circ}$ to $45^{\circ}$ ( $p=0.009$ ), patella shift at $90^{\circ}$ flexion $(\mathrm{p}=0.002)$ and patella tilt at $45^{\circ}$ and $90^{\circ}$ flexion $(\mathrm{p}=0.015$ and $\mathrm{p}=0.005$ respectively).

The results of the CART analysis showed relationships that were not linear in nature. When segmenting the varus/valgus angular change into those with a varus angular change from extension to full flexion between 0 and 4 degrees (long leg axis) and those with either a greater varus change or a valgus change, the knees between $0^{\circ}$ and $4^{\circ}$ had a significantly better KOOS pain score of 7.1 points ( $82.5 \& 89.6$, $\mathrm{p}=0.049$ ). Likewise, measured rollback of no more than $6 \mathrm{~mm}$ without roll forward scored 10.1 points higher ( $79.0 \& 89.1, \mathrm{p}=0.015)$ in the postoperative KOOS pain score. These two groups formed are shown in Figure 3. 

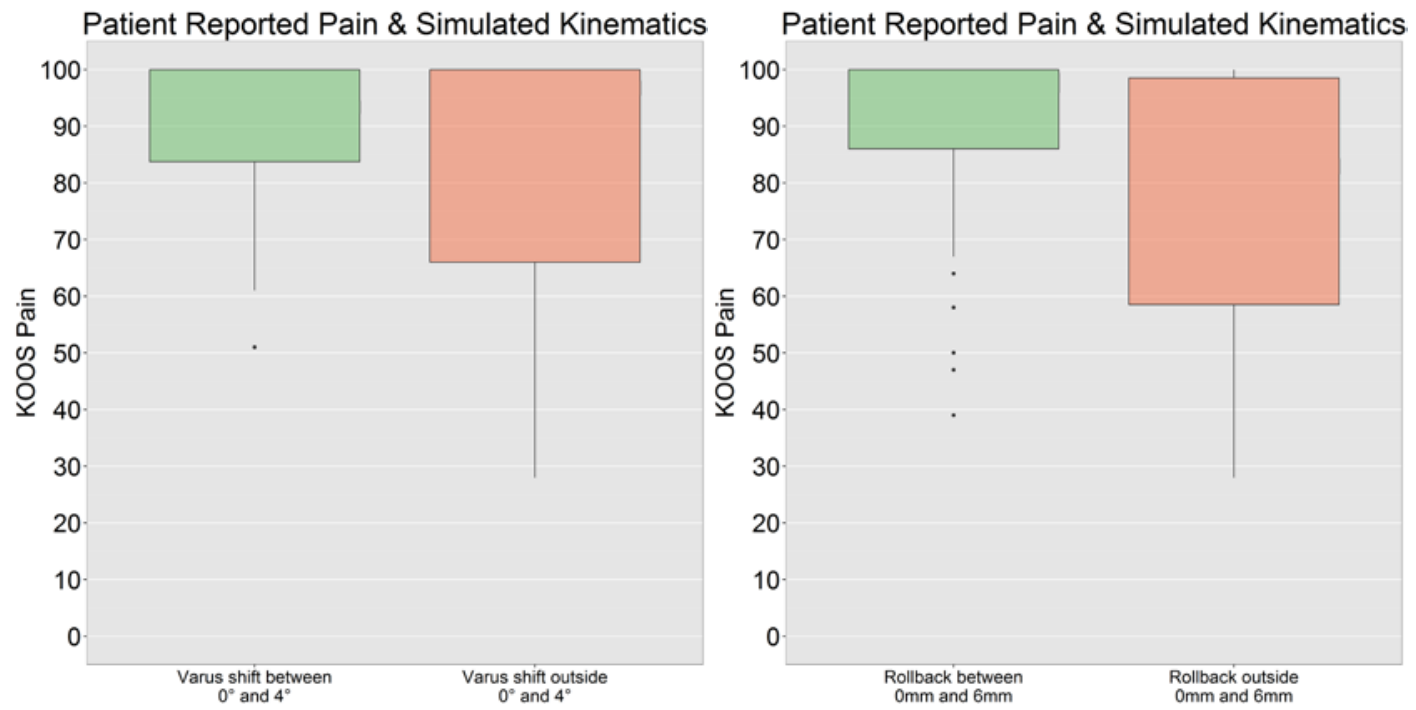

Figure 3:Patient reported postoperative KOOS pain score and a) grouping by shift to varus and b) grouping by rollback within $6 \mathrm{~mm}$.

Table 1 shows the cross correlation table for all dynamic parameters at $90^{\circ}$ of flexion. Correlations are, for the most part, relatively weak, suggesting these parameters are relatively independent of each other. Femoral rollback and varus/valgus have a very low correlation of -0.026 , not significantly different from 0 . This creates the opportunity for these parameters combined to produce a stronger relationship with outcome than they do individually.

\begin{tabular}{|l|r|r|r|r|r|r|}
\cline { 2 - 7 } \multicolumn{1}{c|}{} & $\begin{array}{l}\text { Tibiofemor } \\
\text { al Rotation }\end{array}$ & $\begin{array}{l}\text { Varus/ } \\
\text { Valgus }\end{array}$ & $\begin{array}{l}\text { Femoral } \\
\text { Rollback }\end{array}$ & $\begin{array}{l}\text { Quadriceps } \\
\text { Force }\end{array}$ & $\begin{array}{l}\text { Patella } \\
\text { Lateral } \\
\text { Shift }\end{array}$ & \multicolumn{1}{l|}{$\begin{array}{l}\text { Patella } \\
\text { Lateral Tilt }\end{array}$} \\
\hline $\begin{array}{l}\text { Tibiofemoral } \\
\text { Rotation }\end{array}$ & $-0.331^{*}$ & 0.005 & $0.212^{*}$ & $-0.434^{*}$ & 0.139 \\
\hline Varus/Valgus & $-0.331^{*}$ & & -0.026 & 0.020 & 0.120 & $0.506^{*}$ \\
\hline $\begin{array}{l}\text { Femoral } \\
\text { Rollback }\end{array}$ & 0.005 & -0.026 & & $0.293^{*}$ & -0.008 & -0.086 \\
\hline $\begin{array}{l}\text { Quadriceps } \\
\text { Force }\end{array}$ & $0.212^{*}$ & 0.020 & $0.293^{*}$ & & -0.058 & $0.247^{*}$ \\
\hline $\begin{array}{l}\text { Patella Lateral } \\
\text { Shift }\end{array}$ & $-0.434^{*}$ & 0.120 & -0.008 & -0.058 & & 0.086 \\
\hline $\begin{array}{l}\text { Patella Lateral } \\
\text { Tilt }\end{array}$ & 0.139 & $0.506^{*}$ & -0.086 & $0.247 *$ & 0.086 & \\
\hline
\end{tabular}

Table 1: Spearman's Rho cross correlation table for kinematic parameters across all simulated patients at $90^{\circ}$ of flexion. $(*)$ denotes a statistically significantly different correlation coefficient from 0 to

$$
\mathrm{p}<0.05
$$


Figure 4 shows both of these parameters plotted as the $\mathrm{x}$ (varus shift) and $\mathrm{y}$ (rollback) axes, with each point representing a single patient's combination of these two kinematic parameters. The colour of each point is its postoperative KOOS pain score. There is a clear trend towards a central, dynamic "safe zone", and plotting both of the above relationships defines a region wherein the postoperative KOOS score is 11.5 points higher $(\mathrm{p}=0.013)$.

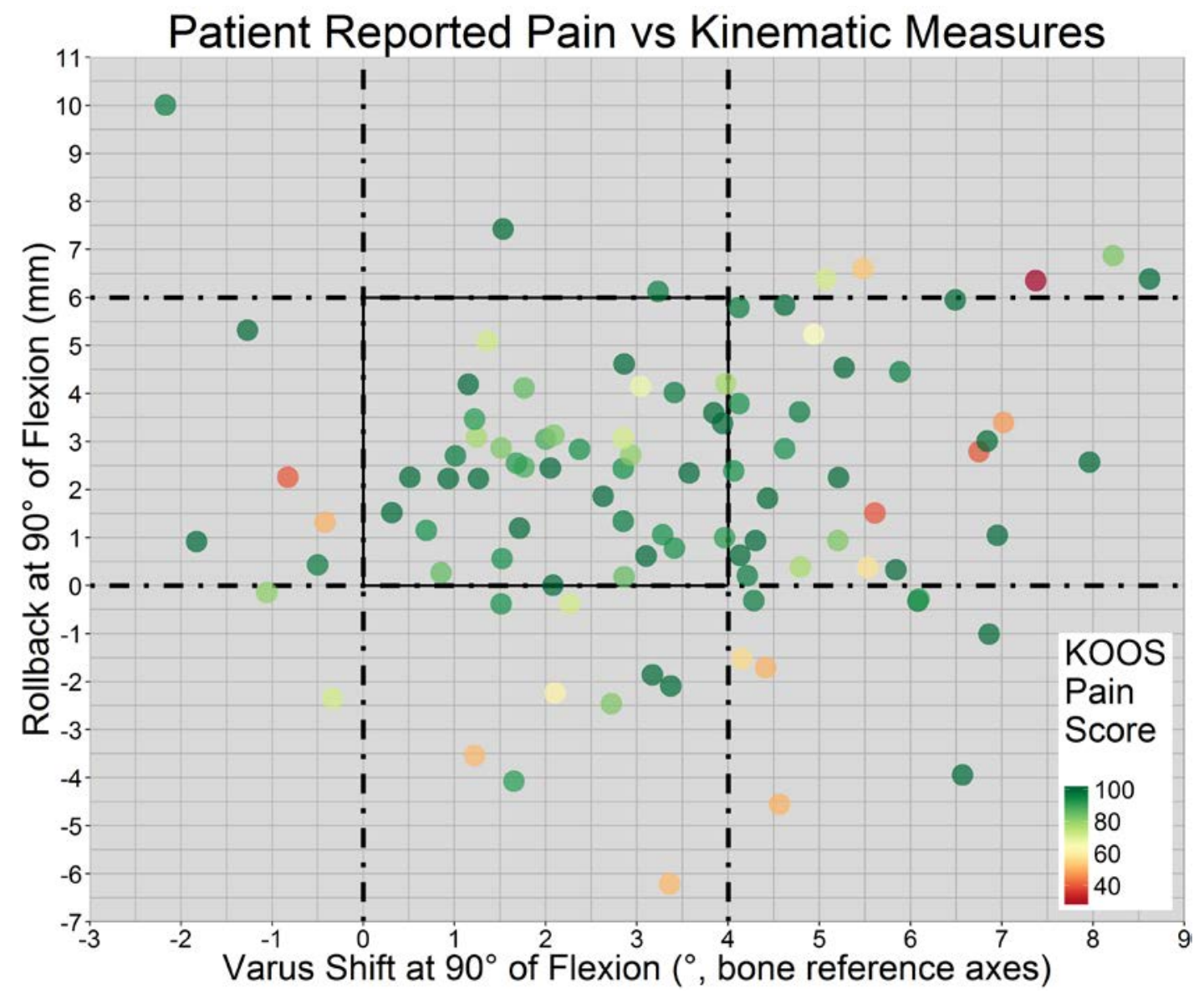

Figure 4: Patient reported pain and rollback, shift to varus plotted against postoperative KOOS pain score

\section{DISCUSSION}

The study showed statistically significant correlations between kinematic factors in a simulated environment of post-operative TKR and post-operative KOOS scores. Relationships between component alignment and kinematic outcomes have been previously shown to exist. [13-16] Results from these studies have historically been used to validate surgical references used in aligning components [30] and inform implant design characteristics. [15, 31] Typically, these have been with reference to kinematic objectives expected to correlate with patient satisfaction or desirable patient 
outcomes, but to the best of the author's knowledge, no previous relationship between a patient reported satisfaction or outcome score and a directly measured kinematic outcome has been shown.

Other alignment parameters have not been shown to have clearly identifiable reproducible relationships with kinematic outcomes, with the result of component alignment and placement producing variable responses from different patients. [17, 18] This might be attributable to variations in patient anatomy [32, 33] and ligamentous constraints. [34] Previous cadaveric studies have shown a relationship between native knee kinematics and lower limb alignment [35] as well as gender. [36] If such relationships can be assumed to impact postoperative dynamics, then component alignment decisions that are responsive to these impacts are desirable.

Table 1 shows the strong independence of the measured dynamic parameters from each other as the knee goes into flexion. The relationships linking each dynamic parameter with component alignment and patient factors are necessarily complex. Simulations are uniquely placed to model the impacts on a patient by patient basis. In vivo tests of widely varied alignment positions are not practical in a routine surgical workflow, while testing options such as in vitro mechanical rig tests or fluoroscopy do not allow for patient specific testing prior to surgery.

One of the limitations of this study is the nature of the dynamic measures reported. The simulation platform used in this study is a multibody model, without the capacity to perform contact stress and deformation calculations seen in other Finite Element models. [19, 26] As a result, rollback has here been measured in terms of the anteroposterior motion of the transepicondylar axis, in order to define a measurement, the combines the simulated motion of the components relative to each other with its implications for the anatomical knee. This hinders interpretability of the results in the context of other studies.

The dynamic factors found in the simulation are the result of both the variation in implantation that occurred and the patient specific, variable musculoskeletal anatomy in which the components were implanted. The presence of a dynamic safe zone in the data suggests a potential patient specific optimisation target for any given individual patient and the opportunity to preoperatively determine a patient specific alignment target to achieve this. 


\section{REFERENCES}

1. Bourne, R.B., et al., Patient satisfaction after total knee arthroplasty: who is satisfied and who is not? Clin Orthop Relat Res, 2010. 468(1): p. 57-63.

2. Beswick, A.D., et al., What proportion of patients report long-term pain after total hip or knee replacement for osteoarthritis? A systematic review of prospective studies in unselected patients. BMJ Open, 2012. 2(1): p. e000435.

3. Baker, P.N., et al., The role of pain and function in determining patient satisfaction after total knee replacement. Data from the National Joint Registry for England and Wales. J Bone Joint Surg Br, 2007. 89(7): p. 893-900.

4. Bullens, P.H., et al., Patient satisfaction after total knee arthroplasty: a comparison between subjective and objective outcome assessments. J Arthroplasty, 2001. 16(6): p. 740-7.

5. Huang, N.F., et al., Coronal alignment correlates with outcome after total knee arthroplasty: five-year follow-up of a randomized controlled trial. J Arthroplasty, 2012. 27(9): p. 1737-41.

6. Nicoll, D. and D. Rowley, Internal rotational error of the tibial component is a major cause of pain after total knee replacement. Journal of Bone \& Joint Surgery, British Volume, 2010. 92(9): p. 1238-1244.

7. Bell, S.W., et al., Component rotational alignment in unexplained painful primary total knee arthroplasty. Knee, 2014. 21(1): p. 272-7.

8. Barrack, R.L., et al., Component rotation and anterior knee pain after total knee arthroplasty. Clin Orthop Relat Res, 2001(392): p. 46-55.

9. Calliess, T., et al., PSI kinematic versus non-PSI mechanical alignment in total knee arthroplasty: a prospective, randomized study. Knee Surg Sports Traumatol Arthrosc, 2016.

10. Dossett, H.G., et al., A randomised controlled trial of kinematically and mechanically aligned total knee replacements: two-year clinical results. Bone Joint J, 2014. 96-B(7): p. 907-13.

11. Wilson, D.A., et al., Changes in the functional flexion axis of the knee before and after total knee arthroplasty using a navigation system. J Arthroplasty, 2014. 29(7): p. 1388-93.

12. Varadarajan, K.M., et al., Can in vitro systems capture the characteristic differences between the flexion-extension kinematics of the healthy and TKA knee? Med Eng Phys, 2009. 31(8): p. 899-906.

13. Tanikawa, H., et al., Influence of Total Knee Arthroplasty on Patellar Kinematics and Patellofemoral Pressure. J Arthroplasty, 2017. 32(1): p. 280-285.

14. Harman, M.K., et al., Prosthesis alignment affects axial rotation motion after total knee replacement: a prospective in vivo study combining computed tomography and fluoroscopic evaluations. BMC Musculoskelet Disord, 2012. 13: p. 206.

15. Carr, B.C. and T. Goswami, Knee implants-Review of models and biomechanics. Materials \& Design, 2009. 30(2): p. 398-413.

16. Werner, F.W., et al., The effect of valgus/varus malalignment on load distribution in total knee replacements. J Biomech, 2005. 38(2): p. 349-55.

17. Bellemans, J., et al., Fluoroscopic analysis of the kinematics of deep flexion in total knee arthroplasty. Influence of posterior condylar offset. J Bone Joint Surg Br, 2002. 84(1): p. 503.

18. Hutter, E.E., et al., Is there a gold standard for TKA tibial component rotational alignment? Clin Orthop Relat Res, 2013. 471(5): p. 1646-53.

19. Ishikawa, M., et al., Kinematic alignment produces near-normal knee motion but increases contact stress after total knee arthroplasty: A case study on a single implant design. Knee, 2015. 22(3): p. 206-12.

20. Dean, S.W., et al., Effects of Patient and Surgical Alignment Variables on Kinematics in TKR Simulation Under Force-Control. Journal of ASTM International, 2006. 3(10): p. 100248. 
21. Victor, J., et al., An experimental model for kinematic analysis of the knee. J Bone Joint Surg Am, 2009. 91 Suppl 6(Supplement 6): p. 150-63.

22. Pianigiani, S., et al., Tibio-femoral kinematics in different total knee arthroplasty designs during a loaded squat: a numerical sensitivity study. J Biomech, 2012. 45(13): p. 2315-23.

23. Kang, K.T., et al., Measuring the effect of femoral malrotation on knee joint biomechanics for total knee arthroplasty using computational simulation. Bone Joint Res, 2016. 5(11): p. 552559.

24. Fitzpatrick, C.K., et al., Relative contributions of design, alignment, and loading variability in knee replacement mechanics. J Orthop Res, 2012. 30(12): p. 2015-24.

25. Mizu-Uchi, H., et al., Patient-specific computer model of dynamic squatting after total knee arthroplasty. J Arthroplasty, 2015. 30(5): p. 870-4.

26. Tanaka, Y., et al., How exactly can computer simulation predict the kinematics and contact status after TKA? Examination in individualized models. Clin Biomech (Bristol, Avon), 2016. 39: p. 65-70.

27. Theodore, W., et al., Variability in Static Alignment and Kinematics for Kinematically Aligned TKA. Knee, 2016 (submitted).

28. Zavatsky, A.B., A kinematic-freedom analysis of a flexed-knee-stance testing rig. J Biomech, 1997. 30(3): p. 277-80.

29. Grood, E.S. and W.J. Suntay, A joint coordinate system for the clinical description of threedimensional motions: application to the knee. J Biomech Eng, 1983. 105(2): p. 136-44.

30. Miller, M.C., et al., Optimizing femoral component rotation in total knee arthroplasty. Clin Orthop Relat Res, 2001(392): p. 38-45.

31. Patil, S., et al., Patient-specific implants with custom cutting blocks better approximate natural knee kinematics than standard TKA without custom cutting blocks. Knee, 2015. 22(6): p. 6249.

32. Patel, A.R., et al., Femoral component rotation in total knee arthroplasty: an MRI-based evaluation of our options. J Arthroplasty, 2014. 29(8): p. 1666-70.

33. Bellemans, J., et al., The Chitranjan Ranawat award: is neutral mechanical alignment normal for all patients? The concept of constitutional varus. Clin Orthop Relat Res, 2012. 470(1): p. 45-53.

34. Ewing, J.A., et al., Estimating patient-specific soft-tissue properties in a TKA knee. J Orthop Res, 2016. 34(3): p. 435-43.

35. Maderbacher, G., et al., Lower Limb Anatomy and Alignment Affect Natural Tibiofemoral Knee Kinematics: A Cadaveric Investigation. J Arthroplasty, 2016. 31(9): p. 2038-42.

36. Varadarajan, K.M., et al., Gender differences in trochlear groove orientation and rotational kinematics of human knees. J Orthop Res, 2009. 27(7): p. 871-8.

\section{DISCLOSURES}

One or more authors are employed or consult to 360 Knee Systems, Sydney. 